\title{
FLORES DE HIBISCO (Hibiscus sabdariffa) COMERCIALIZADAS NO MUNICÍPIO DE CAMPOS DOS GOYTACAZES-RJ: UMA ANÁLISE DO PERFIL FITOQUÍMICO E DO USO POPULAR, CONSIDERANDO ATIVIDADES ATRIBUIDAS ÀS RESPECTIVAS CLASSES DE METABÓLIDOS SECUNDÁRIOS ENCONTRADAS
}

\section{HIBISCO FLOWERS (Hibiscus sabdariffa) COMMERCIALIZED IN THE MUNICIPALITY OF CAMPOS DOS GOYTACAZES-RJ: AN ANALYSIS OF THE PHYTOCHEMICAL PROFILE AND POPULAR USE, CONSIDERING ACTIVITIES ASSIGNED TO THE RESPECTIVE SECONDARY METABOLID CLASSES}

\author{
Ruama Viana de Barros ${ }^{1}$, Virginia Freitas Rodrigues ${ }^{2}$, Sílvia Menezes de Faria Pereira ${ }^{3}$ e Inêz Barcellos de \\ Andrade $^{4}$ \\ ${ }^{1}$ Aluna do Curso de Graduação em Farmácia na Faculdade de Medicina de Campos (FMC). \\ 2 Doutora em Ciências Naturais. Universidade Estadual do Norte Fluminense (UENF). Professora da Faculdade \\ de Medicina de Campos (FMC). \\ ${ }^{3}$ Doutora em Engenharia e Ciência dos Materias.Universidade Estadual do Norte Fluminense (UENF). \\ Professora da Faculdade de Medicina de Campos (FMC). \\ ${ }^{4}$ Doutora em Educação em Ciências e Saúde. Universidade Federal do Rio de Janeiro \\ (UFRJ/NUTES). Professora da Faculdade de Medicina de Campos (FMC).
}

\section{RESUMO}

As formas mais antigas para prevenção de doenças, tratamento e cura, estão na utilização das plantas medicinais. Dentre as várias espécies utilizadas pela população, encontra-se o Hibisco (Hibiscus sabdariffa). Pertencente à família botânica Malvaceae, o Hibisco é um arbusto anual, nativo dos continentes africano e asiático, que se encontra distribuído nas regiões tropicais e subtropicais de ambos os hemisférios, tornando-se naturalizado em muitas áreas das Américas. Estudos têm comprovado o uso de Hibisco como agente diurético, antimicrobiano, laxante, sedativo, antihipertensivo, entre outros. Mais recentemente, há indicativo de que o Hibisco possa agir como antioxidante, antimutagênico, antitumoral e antileucêmico. $O$ presente trabalho teve como objetivo realizar uma análise fitoquímica preliminar nas flores de Hibiscus sabdariffa, comercializadas em Campos dos Goytacazes, RJ, e correlacionar os resultados obtidos com as atividades farmacológicas atribuídas às classes de metabólitos encontrados. O presente estudo revelou a presença de grande quantidade $(52,09 \%)$ de metabólitos de alta polaridade nas flores de Hibisco. Análise

\section{ABSTRACT}

The oldest forms for disease prevention, treatment and cure are in the use of medicinal plants. Among the various species used to the population is Hibiscus (Hibiscus sabdariffa). Belong to the botanical family Malvaceae, Hibiscus is an annual shrub native from African and Asian, which is distributed in the tropical and subtropical regions of both hemispheres, it is becoming naturalized in many areas of the Americas. Studies have proven the use of Hibiscus as a diuretic, antimicrobial, laxative, sedative, antihypertensive and others. Recently, there is indicative that Hibisco can act as antioxidant, antimutagenic, antitumor and antileukemic. The present work aimed to perform a preliminary phytochemical analysis of Hibiscus sabdariffa flowers, commercialized in Campos dos Goytacazes, RJ, and to correlate the results obtained with the pharmacological activities attributed to the secondary metabolic classes. The present study revealed the presence a plenty of high polarity metabolites in Hibiscus flowers (52.09\%). Phytochemical analysis revealed the presence of tannin in the extract flowers from this plant species 
fitoquímica de identificação revelou a presença do metabólito secundário da classe dos taninos, nas flores analisadas da espécie vegetal (Hibiscus sabdariffa). A análise de identificação de flavonoides, por sua vez, não permitiu confirmar a presença desse metabólito visto que a coloração da própria espécie mascarou o resultado. As atividades atribuídas, em referências bibliográficas, à espécie Hibiscus sabdariffa, em especial antimicrobiana e antibiótica, foi compatível com as atividades relacionadas à classe de metabólitos identificada na amostra. Tal estudo revela um resultado preliminar, mas pode nortear outros estudos na busca de substâncias com atividade antioxidante.

Palavras-chaves: Hibiscus sabdariffa, Flavonoides, Taninos.

\section{INTRODUÇÃO}

A utilização de plantas medicinais para prevenção de doenças, tratamento e cura é uma das mais antigas formas de prática medicinal da humanidade. A Organização Mundial de Saúde (OMS) divulgou no início da década de 1990 que 65$80 \%$ da população dos países em desenvolvimento dependiam das plantas medicinais como única forma de acesso aos cuidados básicos de saúde (MACIEL et al, 2002).

A importância das plantas medicinais desde as primeiras civilizações implica diretamente no próprio sustento e cultivo dos alimentos pela população humana, portanto, existe interesse de pesquisadores para realização de estudos envolvendo fontes de medicina natural. Muitos estudiosos têm direcionado suas pesquisas para produtos naturais com análises fitoquímicas através da utilização de espectroscopia UV, uma técnica que define a presença de substâncias que possuem ligações insaturadas em conjugação (MACIEL et al, 2002).

Essas substâncias têm sido utilizadas como antioxidantes naturais que tem aliviado sinais e sintomas de doenças como envelhecimento, câncer, aterosclerose, inflamação, etc, que são consequências de doenças humanas associadas ao estresse oxidativo, que é um excesso de agentes oxidantes e ou deficiência do sistema protetor
(Hibiscus sabdariffa). Flavonoid test did not confirm the presence of this metabolite once the extract coloration has masked the result. As mentioning in the literature data the antimicrobial and antioxidant activities from Hibiscus are attributed the tannis and flavonoids class. The preliminary result in this study can guide other research in the browse of substances with antioxidant and antimicrobial activity.

Keywords: Hibiscus sabdariffa, Flavonoids, Tannins.

(MACIEL et al, 2002).

Os radicais livres, moléculas altamente reativas que contêm número ímpar de elétrons em sua última camada eletrônica que confere alta reatividade a esses átomos ou moléculas devido ao não aparelhamento de elétrons, são formados a partir de reações de oxirredução e produzem efeitos deletérios no organismo (FERREIRA; MATSUBARA,1997).

Muitos estudos têm se voltado para investigação sobre compostos fenólicos devido seus benefícios como redução de doenças cardíacas, redução do colesterol e câncer, com base em atividade antioxidante (VIZOTTO et al, 2009).

A atividade antioxidante de muitas plantas está relacionada à presença dos metabólitos fenólicos. Essas substâncias inibem a formação de radicais livres e têm sido associadas a uma menor incidência de doenças relacionadas com o estresse oxidativo que pode intensificar o risco para o desenvolvimento de várias doenças (PINHO, 2016).

Segundo Gobbo-Neto e Lopes (2007) diversos fatores como: radiação ultravioleta, temperatura, altitude e disponibilidade hídrica podem influenciar qualitativamente na produção dos metabólitos secundários. No tangente a produção de metabólitos fenólicos, como flavonoides e taninos, estudos revelam que há uma correlação positiva entre a intensidade de radiação 
solar e a produção dos mesmos.

Correlação positiva também é descrita entre o conteúdo total de flavonoides e a altitude.

Segundo Sobota (2006), a espécie Hibiscus sabdariffa $L$ tem sido empregada com finalidade antioxidante, sendo as folhas e os cálices as partes mais usadas. Seus cálices (Figura 1) se caracterizam por uma coloração avermelhada devido à presença de flavonoides e um sabor ácido adstringente devido à presença de taninos que é um importante metabólito secundário, atraindo, portanto, as indústrias de alimentos e farmacêuticas.

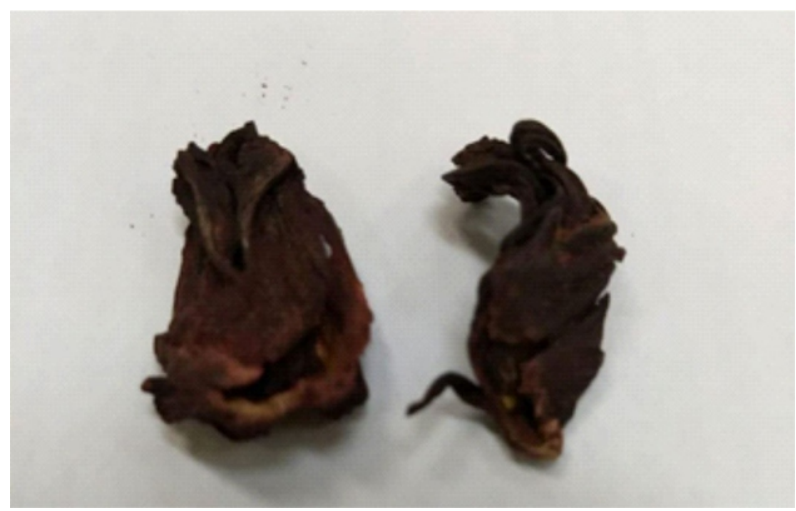

Figura 1: Hibiscus sabdariffa (Foto: BARROS, 2018)

Originária da Índia, do Sudão e da Malásia, pertencente à família Malvaceae e ao gênero Hibiscus, o Hibisco é uma planta herbácea anual de clima seco, comum em região montanhosa subtropical, podendo chegar até $1,5 \mathrm{~m}$ de altura, de caule arroxeado, folhas alternas verde-arroxeadas, flores solitárias, amarelas, axilares, que duram um dia, produz frutos vermelhos do tipo cápsula (SOBOTA; PINHO, 2016).

Suas principais substâncias antioxidantes são polifenóis como flavonoides e taninos (VIZZOTO et al,2009).

\section{TANINOS}

Os taninos são metabolitos secundários, também denominados de metabolitos especiais, fenólicos, de grande interesse econômico e ecológico. Tais compostos são responsáveis pela adstringência de muitos frutos e produtos vegetais (ROCHA et al, 2011).

Grande parte dos compostos fenólicos não é encontrada no estado livre na natureza, mas sob forma de ésteres ou de heterosídeos sendo, portanto, solúveis em água e em solventes orgânicos polares. Os taninos por serem fenólicos são muitos reativos quimicamente, formam pontes de hidrogênio, intra e intermoleculares. São classificados em dois importantes grupos: hidrolisáveis e condensados (PAIVA et al, 2002).

Os taninos hidrolisáveis (Figura 2) consistem de ésteres de ácidos gálicos e elágicos. $\mathrm{O} \quad \mathrm{s}$ condensados (Figura 3) por sua vez, se caracterizam pelos polímeros de fravan-3-ol e/ou fravan 3,4-diol (MONTEIRO et al, 2005).
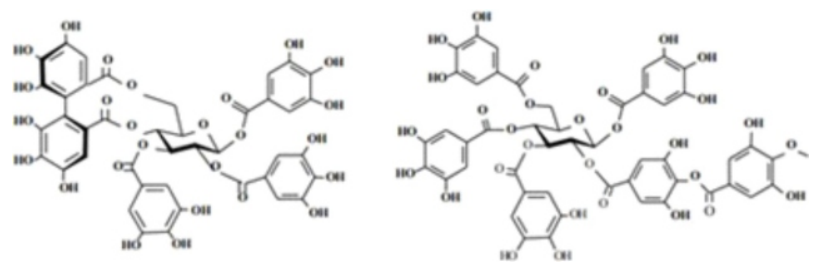

Figura 2: Taninos hidrolisáveis(Fonte: SIMÕES,2007)

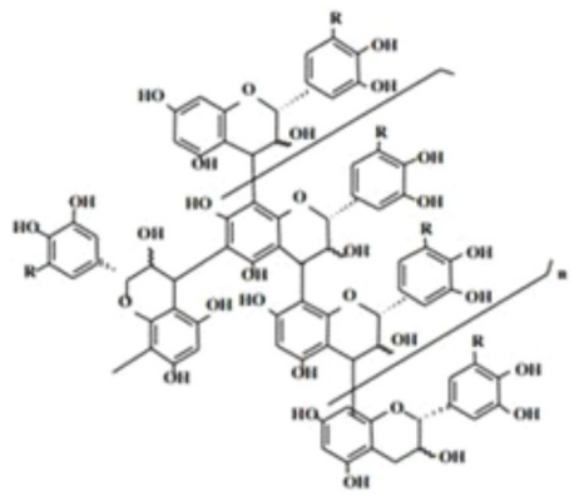

Figura 3: Taninos condensados (Fonte: SIMÕES, 2007)

\section{FLAVONOIDES}

Os flavonoides representam um dos grupos fenólicos mais importantes e diversificados entre os produtos naturais. Eles apresentam um núcleo característico C6-C3-C6, oriundo da rota biossintética do ácido chiquimato e do ácido acético (SIMÕES, 2007) (Figura 4).

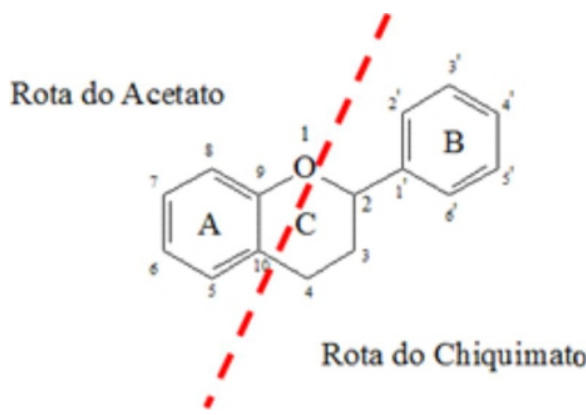

Figura: 4: Estrutura base dos flavonoides

Diversas funções importantes são atribuídas aos 
flavonoides, dentre as quais destacam-se: proteção dos vegetais contra a incidência de raios ultravioleta e visível, proteção contra microrganismos e atividade antioxidante (SIMÕES,2007, MUZITANO, 2009 \&SASKIA et al, 1996).

Grande parte da utilização de flavonoides pela população para tratamento de doenças é empírica pois as ciências química e biológica são muito mais recentes que o conhecimento dessa classe. Entretanto, atualmente, muitos trabalhos têm comprovado, cientificamente, uma diversidade de atividade biológica exercida pelos flavonoides como: analgésica, anticâncer, antianginosa, antiinflamatória e vaso protetor (RODRIGUES, 2013).

Neste contexto, o presente trabalho teve como objetivo realizar uma análise fitoquímica preliminar nas flores de Hibiscus sabdariffa, comercializadas em Campos dos Goytacazes, RJ, e correlacionar os resultados obtidos com as atividades farmacológicas descritas na literatura para as classes de metabólitos encontradas com o uso popular da espécie.

\section{MATERIALE MÉTODO}

Trata-se de um estudo do tipo observacional transversal realizado a partir do extrato metanólico, obtido por maceração, de flores do Hibiscus sabdariff e uma revisão de literatura. A busca por textos publicados de 1996 até 2015 foi realizada na base de dados eletrônico SciELO.

Os ensaios foram desenvolvidos no laboratório de Química da Faculdade de Medicina de Campos (FMC).

Foi realizada análise na pesquisa bibliográfica e obtidos 25 artigos dos quais foram lidos e selecionados 12 para a correlação dos resultados apresentados com os obtidos no presente estudo.

Material vegetal

As flores de Hibiscus sabdariffa foram adquiridas no comércio de Campos dos Goytacazes, RJ.

\section{Obtenção do extrato}

Uma massa de 51,984g de flores secas de Hibiscus sabdariffa foi submetida ao processo de extração única por maceração em metanol, volume em quantidade suficiente para imergir toda a amostra $(240 \mathrm{~mL})$. Após 48 horas, o extrato metanólico obtido, foi filtrado e evaporado até secura em banho-maria (Figura 5).
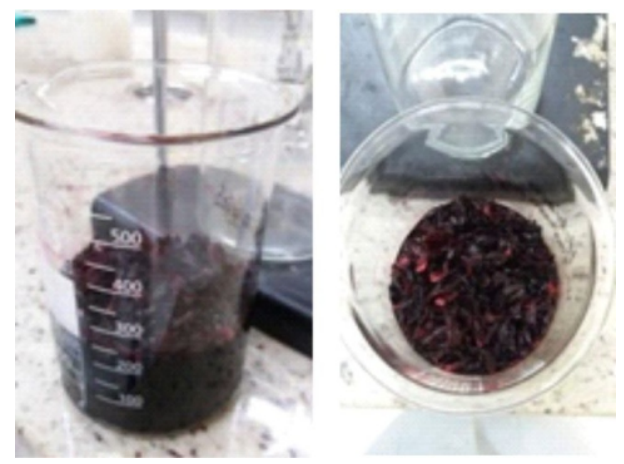

Figura 5 : Extração metanólica das flores de Hibiscus sabdariffa pelo método de maceração (Foto: BARROS, 2018).

\section{ANÁLISES FITOQUÍMICAS}

A análise fitoquímica qualitativa da amostra para verificação da ocorrência dos metabólitos da classe dos Taninos e Flavonoides foi realizada como descrito pela Sociedade Brasileira de Pharmacognosia.

a) Identificação química de Taninos

Extração:

$5 \mathrm{~g}$ de amostra vegetal pulverizada foi submetida a extração por decocção com $100 \mathrm{ml}$ de água destilada.

Os extratos obtidos foram filtrados e distribuídos em 4 tubos de ensaio, numerados de 1 a 4.

\section{TESTES DE IDENTIFICAÇÃO}

Para esta metodologia, duas técnicas dando reação positiva confirmam a presença de taninos.

Tubo 1- Gelatina. Foram transferidos $2 \mathrm{ml}$ de filtrado para o tubo 1 , acrescidos de duas gotas de ácido clorídrico, mais solução de gelatina a $2,5 \%$ gota a gota.

*Se ocorrer formação de precipitado: reação positiva para taninos.

Tubo 2- Cloreto férrico. Foram transferidos $2 \mathrm{ml}$ do filtrado para o tubo 2, mais $10 \mathrm{ml}$ de água destilada somado de 2 a 4 gotas da solução de cloreto férrico a $1 \%$ em metanol.

* Cor Azul: taninos hidrolisáveis ou gálico

Cor Verde: taninos condensados ou catéquico

Tubo 3- Acetato de chumbo. Adicionou-se $5 \mathrm{ml}$ do filtrado no tubo 3 , acrescidos de $10 \mathrm{ml}$ da solução de 
ácido acético a $10 \%$, mais $5 \mathrm{ml}$ da solução de acetato de chumbo $10 \%$.

*Formação de um precipitado esbranquiçado: presença de taninos hidrolisáveis.

Tubo 4- Branco (extrato)

Observação: Duas técnicas dando positiva confirmam a presença de taninos.

b) Identificação química de Flavonoides

\section{Extração:}

1g da amostra vegetal foi fervida em banho-maria com $10 \mathrm{ml}$ de solução de Etanol (70\%) por 2 minutos e, em seguida, realizou-se a filtração.

Testes de identificação: Reação de Shinoda

Transferiu-se cerca de $2 \mathrm{ml}$ de extrato alcoólico para um tubo de ensaio e acrescentou-se mais ou menos seis fragmentos de Mg metálico. Em seguida, adicionou-se $1 \mathrm{ml}$ de $\mathrm{HCl}$ concentrado.

*Pesquisa positiva-coloração rósea a vermelha

\section{RESULTADOSEDISCUSSÃO}

$O$ processo de maceração das flores em metanol obteve um rendimento de $27,08 \mathrm{~g}(52,09 \%)$ de extrato seco, o que aponta para ocorrência de uma quantidade significativa de substâncias de alta polaridade nessa parte do vegetal.

A análise fitoquímica das flores analisadas da espécie vegetal (Hibiscus sabdariffa) revelou resultado positivo para taninos visto que o tubo 2 observou- se a cor azul, confirmando a presença de taninos do tipo hidrolisáveis ou gálico e no tubo 3 observou-se a ocorrência da formação de precipitado esbranquiçado (Figura 6). Embora o tubo 1 não tenha apresentado formação de precipitado o resultado de identificação da presença de taninos é válido pois, segundo a referência, duas técnicas dando reação positiva confirmam a presença de taninos.

De acordo com Monteiro et al (2005), a sazonalidade é um fator responsável pela variação quantitativa de taninos em drogas vegetais.

A análise de identificação de flavonoides, por sua vez, não revelou alteração de cor quando compara com o controle, o que não caracteriza presença desse metabólito na amostra (Figura 7).

Tal resultado, entretanto, pode ter sido comprometido pela cor original do extrato já ser vermelha.

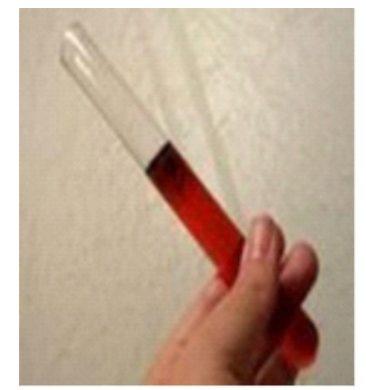

Tubo 2

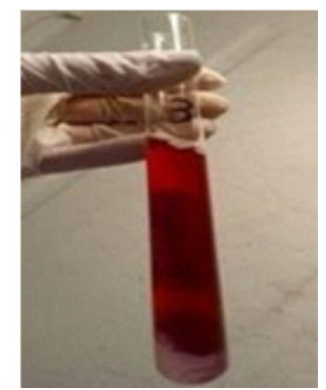

Tubo 3
Figura 6: Resultado do teste de identificação de taninos. (Foto: BARROS, 2018)

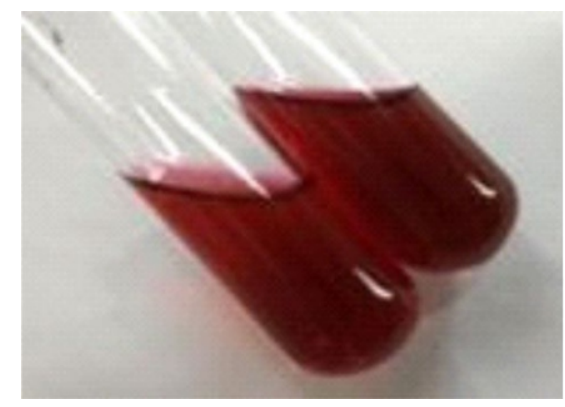

Figura 7:Resultado do teste de identificação de flavonoides (Foto: BARROS, 2018)

O estresse hídrico é um fator a ser levado em consideração frente ao resultado preliminar obtido para presença de flavonoides na espécie, visto que os efeitos da chuva podem alterar a produção dos metabólitos secundários, nem sempre atuando de forma positiva quando em excesso.

Segundo Gobbo-Neto e Lopes (2007), a correlação positiva entre a intensidade de radiação solar e a produção de metabólitos como flavonoides e taninos proporciona aumento nos níveis de defesa antioxidante do vegetal. Tal consideração ratifica a importância da atividade dos fenólicos para o vegetal e o uso da espécie como antioxidante pela população.

Os dois fatores supracitados (estresse hídrico e radiação solar) estão diretamente relacionados com a estação do ano, entretanto, não havia na embalagem informações referentes à data de coleta das flores de Hibiscus sabdariffa analisadas.

Outras correlações entre a utilização da espécie Hibiscus sabdariffa e as atividades atribuídas às classes de taninos e flavonoides, com base na literatura, estão listados no quadro a seguir (Quadro $1)$. 
Quadro 1- Atividades atribuídas aos flavonoides e taninos

Quadro 1- Atividades atribuidas aos flavonoides e taninos

\begin{tabular}{|c|c|c|c|c|}
\hline Ano & Referências & Flavonoides & Taninos & $\begin{array}{l}\text { Atividades } \\
\text { atribuidas }\end{array}$ \\
\hline 1996 & $\begin{array}{l}\text { ACKER, S.A.B.E.V.; } \\
\text { et al. Structual } \\
\text { aspects of } \\
\text { antioxidant activity of } \\
\text { flavonoids. Elsevier }\end{array}$ & $\begin{array}{l}\text { Presença de } \\
\text { Flavonoide }\end{array}$ & & $\begin{array}{c}\text { Atividade } \\
\text { Antioxidante }\end{array}$ \\
\hline 2002 & $\begin{array}{l}\text { HAVASTEEN, H } \\
\text { B.The } \\
\text { Biochemistry and } \\
\text { medical significance } \\
\text { of the flavonoids. } \\
\text { Elsevier }\end{array}$ & $\begin{array}{l}\text { Presença de } \\
\text { Flavonoide }\end{array}$ & & $\begin{array}{c}\text { Atividade } \\
\text { Antioxidante }\end{array}$ \\
\hline 2002 & $\begin{array}{l}\text { PAIVA, S.R.; } \\
\text { HERINGER, A.P.; } \\
\text { FIGUEIREDO, M.R.; } \\
\text { KAPLAN, M.A.C. } \\
\text { Taninos } \\
\text { condensados de } \\
\text { espécies de } \\
\text { Plumbaginaceae. Re } \\
\text { v. Floresta e } \\
\text { ambiente }\end{array}$ & & $\begin{array}{c}\text { Presença de } \\
\text { Taninos }\end{array}$ & $\begin{array}{c}\text { Atividade } \\
\text { Antioxidante }\end{array}$ \\
\hline 2005 & $\begin{array}{l}\text { MONTEIRO, J.M. et } \\
\text { al. Taninos: uma } \\
\text { abordagem da } \\
\text { química à ecologia. } \\
\text { Rev. Quim Nova }\end{array}$ & & $\begin{array}{l}\text { Presença de } \\
\text { Taninos }\end{array}$ & $\begin{array}{c}\text { Por via interna } \\
\text { exercem efeito } \\
\text { antidiarreico e anti- } \\
\text { séptico. Por via } \\
\text { externa } \\
\text { impermeabilizam } \\
\text { as camadas mais } \\
\text { expostas da pele e } \\
\text { mucosas, } \\
\text { protegendo assim } \\
\text { as camadas } \\
\text { subjacentes. Ao } \\
\text { precipitar } \\
\text { proteinas, os } \\
\text { taninos propiciam } \\
\text { um efeito } \\
\text { antimicrobiano e } \\
\text { antifúngico. }\end{array}$ \\
\hline 2008 & $\begin{array}{l}\text { MACHADO, H.; } \\
\text { NAGEM, T.J.; } \\
\text { PETERS, V.M., } \\
\text { FONSECA, C.S.; } \\
\text { OLIVEIRA, T.T. } \\
\text { Flavonoides e seu } \\
\text { potencial } \\
\text { terapêutico. Boletim } \\
\text { do Centro de } \\
\text { Biologia da } \\
\text { Reprodução. }\end{array}$ & $\begin{array}{c}\text { Presença de } \\
\text { Flavonoide }\end{array}$ & & $\begin{array}{c}\text { Atividades } \\
\text { Antioxidantes; } \\
\text { Atividades anti- } \\
\text { inflamatórias; } \\
\text { Inibição de ciclo } \\
\text { celular. }\end{array}$ \\
\hline 2009 & $\begin{array}{l}\text { COUTINHO,M.A.S.; } \\
\text { MUZITANO, } \\
\text { M.F.;COSTA, S.S. } \\
\text { Flavonoides: } \\
\text { Potenciais Agentes } \\
\text { Terapêuticos para o } \\
\text { Processo } \\
\text { Inflamatório. Rev. } \\
\text { Virtual de Quim. }\end{array}$ & $\begin{array}{l}\text { Presença de } \\
\text { Flavonoide }\end{array}$ & & $\begin{array}{c}\text { Atividade } \\
\text { antinflamatória. }\end{array}$ \\
\hline 2011 & $\begin{array}{l}\text { BERNARDES, N.R.; } \\
\text { et al. Quantificação } \\
\text { dos teores de } \\
\text { Taninos e fenóis } \\
\text { totais e Avaliação da } \\
\text { Atividade } \\
\text { Antioxidante dos } \\
\text { Frutos de Aroeira. } \\
\text { Rev. Vértices }\end{array}$ & $\begin{array}{l}\text { Presença de } \\
\text { Flavonoide }\end{array}$ & $\begin{array}{c}\text { Presença de } \\
\text { Taninos }\end{array}$ & $\begin{array}{c}\text { Atividade } \\
\text { Antioxidante. }\end{array}$ \\
\hline 2012 & $\begin{array}{l}\text { POZZAN, M.S.V.; } \\
\text { BRAGA, G.C.: } \\
\text { SALIBE, A.B. Teores } \\
\text { de antocianinas, } \\
\text { fenóis totais, taninos } \\
\text { e ácido ascórbico } \\
\text { em uva 'bordô'' sobre } \\
\text { diferentes porta- } \\
\text { enxertos. Rev. } \\
\text { Ceres }\end{array}$ & $\begin{array}{l}\text { Presença de } \\
\text { Flavonoide }\end{array}$ & $\begin{array}{c}\text { Presença de } \\
\text { Taninos }\end{array}$ & $\begin{array}{c}\text { Atividade } \\
\text { Antioxidante. }\end{array}$ \\
\hline 2014 & $\begin{array}{l}\text { ALMEIDA, T.D.; MAI, } \\
\text { B.F.; PUGET, F.P. } \\
\text { EXTRAÇAO DE } \\
\text { TANINO DA CASCA } \\
\text { DO CAFE. Rev. } \\
\text { Cobec }\end{array}$ & & $\begin{array}{c}\text { Presença de } \\
\text { Taninos }\end{array}$ & $\begin{array}{c}\text { Atividade } \\
\text { Antioxidante. }\end{array}$ \\
\hline 2015 & $\begin{array}{l}\text { PEREIRA, A. V et al. } \\
\text { Taninos da casca do } \\
\text { Cajueiro: atividade } \\
\text { antimicrobiana. Rev. } \\
\text { Agrotec }\end{array}$ & & $\begin{array}{c}\text { Presença de } \\
\text { Taninos }\end{array}$ & $\begin{array}{c}\text { Atividade } \\
\text { Antimicrobiana }\end{array}$ \\
\hline
\end{tabular}

De acordo com Coutinho et al (2009) e Machado et al (2008) encontraram em análises fitoquímicas atividades anti-inflamatórias tanto para flavonoides quanto para taninos.

Segundo Machado et al (2009), Moline et al (2000), Havsteen et al (2002), Bernardes et al (2011), Pozzan et al (2012), Almeida et al (2014), Acker et al (1996) e Paiva et al (2008), obtiveram em suas análises fitoquímicas atividade antioxidantes para os taninos e flavonoides, sendo a atividade mais destacada nesta revisão de literatura.

Para atividade antimicrobiana Monteiro et al (2005) e Pereira et al (2015) tiveram resultados relevantes para taninos e flavonoides.

Diante do exposto, a correlação dos resultados obtidos através do levantamento das atividades descritas na literatura para os metabólitos da classe dos flavonoides e taninos vão ao encontro da utilização das flores de Hibiscus sabdariffa, objetivo deste trabalho, uma vez que apresentam atividade condizente com o uso.

\section{CONCLUSÃO}

O presente estudo revelou a presença majoritária de metabólitos de alta polaridade nas flores de Hibisco.

Pelas análises fitoquímicas preliminares, realizadas nas flores da espécie, e pelo levantamento bibliográfico realizado sobre atividade atribuída a taninos e flavonoides, é possível correlacionar as atividades atribuídas à espécie Hibiscus sabdariffa com as classes de metabólitos especiais citadas, em especial os taninos, classe de metabólitos identificada na amostra.

Os resultados obtidos e analisados no estudo, ao confirmar a presença de metabólitos fenólicos apontam para uma utilização compatível das flores de Hibiscus sabdariffa pela população, principalmente no tangente à propriedade antioxidante.

Embora preliminares, os resultados obtidos neste estudo podem nortear outras pesquisas na busca de novas drogas e confirmação de outras atividades, com realização de isolamento, identificação de substâncias e ensaios biológicos com a espécie. 


\section{REFERÊNCIASBIBLIOGRÁFICAS}

ACKER, S.A.B.E.V., et al. Structural aspects of antioxidant activity of flavonoids.

Elsevier. v.20, p.331-342, 1996

ALMEIDA, T.D.; MAI, B.F.; PUGET, F.P. EXTRAÇÃO DE TANINO DA CASCA DO

CAFÉ. Rev. Cobec. Florianópolis, vol. 1 n. 2, 2014

BERNARDES, N.R.; et al. Quantificação dos teores de Taninos e fenóis totais e Avaliação da Atividade Antioxidante dos Frutos de Aroeira. Rev. Vértices. Campos dos Goytacazes, , v. 13, n. 3, p. 117-128, set./dez. 2011ACKER, S.A.B.E.V., et al. Structural aspects of antioxidant activity of flavonoids. Elsevier. v.20, p.331-342, 1996

ALMEIDA, T.D.; MAI, B.F.; PUGET, F.P. EXTRAÇÃO DE TANINO DA CASCA DO

CAFÉ. Rev. Cobec. Florianópolis, vol. 1 n. 2, 2014

BERNARDES, N.R.; et al. Quantificação dos teores de Taninos e fenóis totais e Avaliação da Atividade Antioxidante dos Frutos de Aroeira. Rev. Vértices. Campos dos Goytacazes, , v. 13, n. 3, p. 117-128, set./dez. 2011

ACKER, S.A.B.E.V., et al. Structural aspects of antioxidant activity of flavonoids.

Elsevier. v.20, p.331-342, 1996

ALMEIDA, T.D.; MAI, B.F.; PUGET, F.P. EXTRAÇÃO DE TANINO DA CASCA DO

CAFÉ. Rev. Cobec. Florianópolis, vol. 1 n. 2, 2014

BERNARDES, N.R.; et al. Quantificação dos teores de Taninos e fenóis totais e Avaliação da Atividade Antioxidante dos Frutos de Aroeira. Rev. Vértices. Campos dos Goytacazes, , v. 13, n. 3, p. 117-128, set./dez. 2011

ARAÚJO, P.B.M.; BRANDÃO, M.S.; CHAVES, M.H. Fenóis Totais e atividade antioxidade de cinco plantas medicinais. Rev. Quim Nova. Piauí, v. 30, 2007

COUTINHO, M.A.S.; MUZITANO, M.F.; COSTA, S.S. Flavonoides: Potenciais Agentes Terapênticos para o Processo Inflamatório. Rev. Virtual de Quim. RJ, v.1, n.3, p. 241-256, Jun 2009

FERREIRA, A.L.A.; MATSUBARA, L.S.. Radicais livres: conceitos, doenças relacionadas, sistema de defesa e estresse oxidativo. Rev. Assoc. Med. Bras. São Paulo , v. 43, n. 1,p. 61-68, Mar. 1997

GOBBA-NETO, L.; LOPES, P.N. Plantas medicinais. Fatores de influência no conteúdo de metabólicos secundários. Quim Nova. São Paulo, v.30, n.2, p. 374- 375, 2007

HAVSTEEN, H.B. The biochemistry and medical significance of the flavonoids.

Elsevier. V.96 $67-202,2002$

MACHADO, H.; NAGEM, T.J.; PETERS, V.M., FONSECA, C.S.; OLIVEIRA, T.T.

Flavonoides e seu potencial terapêutico. Boletim do Centro de Biologia da Reprodução. Juiz de Fora, v. 27, n. 1/2, p. 33-39, 2008

MACIEL, Maria Aparecida M. et al . Plantas medicinais: a necessidade de estudos multidisciplinares. Quim Nova,SãoPaulo, v. 25, n. 3, p. $429-438$,

May. 2002

MONTEIRO, J.M. et al . Taninos: uma abordagem da química à ecologia.Quim Nova.

São Paulo, v. 28, n. 5, p. 892-896, Oct. 2005

MOLINE, J.; BUKHAROVICH, M.S .; WOLFF, R.P. Dietary flavononoids and hypertension is there a link? Rev. Medical hypotheses. New York, v. 55 (4), 306- 309, 2000

PAIVA S.R.; HERINGER, A.P.; FIGUEIREDO, M.R.; KAPLAN, M.A.C. Taninos

condensados de espécies de plumbaginaceae. Rev. Floresta e ambiente. RJ. Jan/Dez 2002

PEREIRA, A. V et al. Taninos da casca do Cajueiro: atividade antimicrobiana. Rev. Agrotec. V. 36, n. 1, p. 121-127, 2015

PINTO, A.C.; Valdir F.; VEIGA Junior .; MACIEL, Maria Aparecida M Plantas medicinais: cura segura ?. Quim. Nova, São

Paulo, v. 28, n. 3, p. 519-528, Junho de 2005

POZZAN, M.S.V.; BRAGA, G.C.; SALIBE, A.B. Teores de antocianinas, fenóis totais, taninos e ácido ascórbico em uva 'bordô' sobre diferentes porta-enxertos. Rev. Ceres. Viçosa, v. 59, n.5, p. 701-708, set/out, 2012

ROCHA, W.S.; LOPES, R.M.; SILVA, D.B.; VIEIRA R.F; SILVA, V.P.; COSTA,

T.S.A. Compostos fenólicos totais e taninos condensados em frutos nativos do cerrado. Rev. Bras. Frutic. SP. Dez 2011

RODRIGUES, V.F. Estudo químico da espécie Trichilia quadrijuga e abordagem químico-biológica da planta medicinal

Cajanus cajan. 2013. Tese (Doutorado em Ciências Naturais) - Universidade Estadual do Norte Fluminense (UENF) Campos dos Goytacazes

SIMÕES, C.M.O. Farmacognosia: da planta ao medicamento. 6a .ed. Porto Alegre, RS: Ed. da UFRGS, 2007. $1102 \mathrm{p}$ 